\title{
The neural circuit for tone-specific plasticity in the auditory system elicited by conditioning
}

\author{
Nobuo Suga ${ }^{1}$ \\ Department of Biology, Washington University, St. Louis, Missouri 63130, USA
}

Auditory fear conditioning elicits plastic changes specific to a conditioning tonal stimulus (CS) in the central auditory system. Gao and Suga (1998) proposed the neural circuit for producing these tone-specific changes, represented by best frequency (BF) shifts. The Gao-Suga model (Fig. 1, solid arrows), elaborated upon by Suga et al. (2000, 2002), states that small (or subthreshold) short-term cortical and collicular BF shifts specific to tone bursts (CS) are evoked by the neural circuit within the auditory cortex (Chowdhury and Suga 2000; Sakai and Suga 2001, 2002; Xiao and Suga 2002; Yan and Ehret 2002; Ma and Suga 2003, 2004) and corticofugal feedback loops (Gao and Suga 1998, 2000) activated by CS alone, and this cortical BF shift is augmented and changed into the long-term BF shift by acetylcholine released into the auditory cortex from the cholinergic basal forebrain, the nucleus basalis (Bakin and Weinberger 1996; Bjordahl et al. 1998; Kilgard and Merzenich 1998; Ma and Suga 2003; Yan and Zhang 2005; Zhang et al. 2005). In this model, the nucleus basalis is activated by the auditory and somatosensory cortices (Gao and Suga 1998, 2000; Ma and Suga 2001, 2003; Ji and Suga 2007b) via the association cortex and amygdala where CS is associated with an unconditioned leg stimulus (US). In addition, CS-US association may also occur in the association cortex. The collicular BF shift is increased by the augmented cortical BF shift through corticofugal feedback and contributes to the development of the large long-term cortical BF shift (Ji et al. 2001; Ma and Suga 2005). The ascending and descending (corticofugal) systems form positive feedback loops for the BF shifts. The gain of these feedback loops is presumably controlled by the thalamic reticular nucleus. (For simplicity, the corticothalamic projection is not described in the Gao-Suga model.) This model is fundamentally different from the Weinberger model (Weinberger 1998, 2007). It proposes that the small, short-lasting cortical BF shift is evoked by the neural net intrinsic to the central auditory system without CS-US association, whereas the Weinberger model proposes that it is evoked by the multisensory thalamic nuclei (the medial division of the medial geniculate body [MGBm] and the posterior interlaminar nucleus [PIN]) only after CS-US association occurs in these nuclei. Many neurophysiological findings indicate that the cortical and collicular BF shifts are evoked without CS-US association in the MGBm and PIN (Bakin and Weinberger 1996; Bjordahl et al. 1998; Kilgard and Merzenich 1998; Chowdhury and Suga 2000; Ma and Suga 2001, 2003, 2005; Sakai and Suga 2001, 2002; Xiao and Suga 2002; Yan and Ehret 2002; Yan and Zhang 2005; Zhang and Suga 2005; Zhang et al. 2005; Wu and Yan 2007) and that the inferior colliculus shows conditioningelicited BF shifts (Gao and Suga 1998, 2000; Ji et al. 2001, 2005; Ji and Suga 2003, 2007b). The Gao-Suga model is based on recent neurophysiological findings (Suga and Ma 2003), whereas the Weinberger model (Weinberger 2007, Fig. 12) does not incorporate any of these findings. Therefore, it is incorrect or incomplete. Suga does not doubt that MGBm neurons show responses to both CS

\section{'Corresponding author.}

E-mail suga@biology.wustl.edu; fax (314) 935-4432.

Article is online at http://www.learnmem.org/cgi/doi/10.1101//m.791408. (tone) and US (foot shock), BF shifts, and long-term potentiation or that they project to layer I in the auditory cortex (Weinberger 2007). However, the MGBm activation and inactivation experiments have not yet been performed to prove that the MGBm evokes the cortical BF shift. The serious problem is Weinberger's interpretation of the role of the MGBm in evoking the cortical BF shift. Suga (2008) hypothesizes that the MGBm is involved in the nonspecific plasticity (nonspecific augmentation) of cortical neurons elicited by unpaired CS and US (Fig. 1, dashed arrows), not in the tone-specific plasticity (BF shift) elicited by paired CS and US.

A conditioned big brown bat shows body movements (Gao and Suga 1998) and a heart-rate decrease (Fig. 2; Ji and Suga 2007a) to the conditioned tonal stimulation (CS). The noticeable cortical and collicular BF shifts are evoked neither by short acoustic stimulation (CS) alone nor by electric leg stimulation (US) alone but by paired stimulation (CS-US). Backward conditioning (US-CS) does not evoke these BF shifts. The BF shifts are specific to the frequency of the CS. Unlike the collicular BF shift, the cortical BF shift gradually increases and reaches a plateau after the termination of the conditioning. This plateau is sustained for a long time (Gao and Suga 2000; Suga et al. 2000; Ji et al. 2001). This long-term cortical BF shift is elicited by an increase in the cortical acetylcholine level resulting from activation of the nucleus basalis (Ji et al. 2001; Ji and Suga 2003; Ma and Suga 2003). Therefore, all these findings of the cortical BF shift in the big brown bat are the same as the findings in rodents and meet "all the criteria for physiological plasticity induced by the associative process" proposed by Weinberger (2004).

\section{Responses to Weinberger's comments on the Gao-Suga model}

Weinberger misrepresents the Gao-Suga model by stating that (1) this model ignores the MGBm completely and proposes that the association cortex is the locus of CS-US convergence, and that (2) the Gao-Suga model asserts that plasticity that develops in the association cortex is relayed (via the amygdala) to the inferior colliculus (Weinberger 2007). Then, he discredits Suga and collaborators' works by the following six comments:

1. About the somatosensory cortex, Weinberger wrote, "Suga and coworkers have relied on findings from cortical inactivations using muscimol.... However, radiotracing studies of muscimol diffusion, using smaller doses than used in these studies, have revealed a considerably more extensive spread (Edeline et al. 2002)." The pair of inactivation (Gao and Suga 2000) and activation (Ma and Suga 2001, 2003) experiments of the somatosensory cortex clearly indicates that the somatosensory cortex plays an important role in evoking the conditioning-elicited BF shifts and that muscimol applied to the somatosensory cortex did not at all affect the responses of the cortical auditory neurons (Fig. 3). To compare the results reported in two different papers, he should understand the dif- 


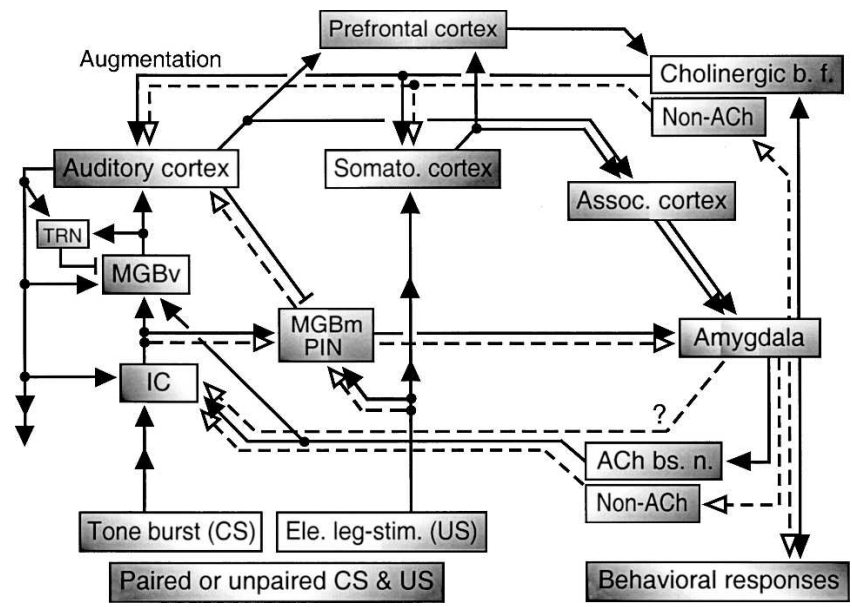

Figure 1. Simplified neural circuits for tone-specific plasticity (BF shifts) elicited by paired CS-US and for nonspecific plasticity (nonspecific augmentation) elicited by unpaired CS-US (working hypothesis). Solid arrows indicate the neural circuit for BF shifts elicited by paired CS-US (conditioning). Dashed arrows indicate the neural circuit for nonspecific augmentation, including the broadening of tuning, elicited by unpaired CS-US (pseudoconditioning). ACh, acetylcholine; b.f., basal forebrain (nucleus basalis); bs. n., brainstem nucleus; CS, conditioned stimulus (tone bursts); IC, inferior colliculus; MGBm, medial division of the medial geniculate body; MGBv, ventral division of the medial geniculate body; PIN, posterior intralaminar nucleus in the thalamus; TRN, thalamic reticular nucleus; US, unconditioned stimulus (electric leg-shock); Arrows, excitatory projections; Projection with a short bar at the end, inhibitory projection. An identical CS tone evokes tone-specific or nonspecific changes in the central auditory system depending on whether it is paired or unpaired with an US. When the cortical BF shift is evoked by the cortical neural net, corticofugal feedback and cholinergic basal forebrain, the auditory cortex probably reduces the signals from the MGBm and PIN which evoke the nonspecific augmentation (solid line with a bar at the end). The cholinergic system augments the BF shift, whereas a noncholinergic neuromodulatory system augments the nonspecific augmentation evoked by the signals from the MGBm and PIN. For simplicity, the neural circuit for eliciting the nonspecific augmentation of subcortical auditory neurons is not included in this model.

ference in experimental methods between them and the data shown in our paper.

2. About the thalamic reticular nucleus, Weinberger wrote, “... this positive feedback loop is turned off by the thalamic reticular nucleus (TRN), which prevents auditory information from reaching AI. ... [They] seem to ignore the fact that the auditory cortex of conditioned animals continues to receive perfectly fine auditory information." This is an overly simplistic interpretation of the role of the reticular nucleus. We never wrote that the thalamic reticular nucleus acts as a simple on/off switch. Villa et al. (1991) suggested that "the corticothalamic projection, [including both direct and indirect projections through the reticular nucleus], may be the support for selective operations such as an adaptive filtering of the incoming acoustic signal at the thalamic level adjusted as a function of cortical activity." I agree with them.

3. About the BF shifts evoked by long repetitive acoustic simulation, Weinberger wrote, "Finally, Suga and col- leagues found that repeated presentation of a tone alone produced increased response to and tuning shifts toward the repeated frequency (Gao and Suga 1998). This is in direct opposition to the extensive literature on the effects of repeated auditory stimulation, which produces response decrements (habituation) in the auditory cortices in other taxa." The cortical and collicular BF shifts were measured by changing the frequency of a tone burst several minutes after, not during, the 30-min-long repetitive tonal stimulation and were observed at a frequency $\sim 5 \mathrm{kHz}$ higher than, not the same as, the frequency of the long repetitive tonal stimulation. Therefore, habituation did not interfere with the BF shifts. As far as I know, no one has performed any experiment on non-bat species similar to or the same as ours.

4. About the medial division of the medial geniculate body (MGBm or MGm), Weinberger wrote, "Why Suga and colleagues ignore the MGm is baffling." My response to this comment is in the last eight lines of the first paragraph of "Background."

5. Weinberger wrote, "Interestingly, studies in the big brown bat (Eptesicus fuscus) have reported CS-specific tuning shifts during tone-shock pairing (Suga and Ma 2003). However, they also lack behavioral validation of association, although, in this case, behavioral data were said to have been obtained ..." My response to this comment is in the second paragraph of "Background."

6. Finally, Weinberger wrote, "Perhaps as an echolocating animal, the principles of auditory system plasticity are specialized in the big brown bat, and thus, it is not a good model for general mammalian auditory associative plasticity." The BF shifts evoked by focal electric stimulation of the auditory cortex (Yan and Ehret 2001, 2002; Sakai and Suga 2002; Ma and Suga 2004) and the neurophysiological properties of the cortical BF shift evoked by auditory fear conditioning are the same for both the big brown bat and rodents. (See the second paragraph of "Background.") Furthermore, muscarinic acetylcholine receptors in the auditory cortex play an essential role in producing the large long-lasting cortical BF shift in the bat and rodents. The BF shift is not a plastic change which is specifically related to echolocation. There is no reason to doubt that the neural circuit for the BF shift is shared with bats and non-bat mammals.

The Weinberger model is simple and straightforward, so one may consider that it is reasonable. However, one should open his/her eyes to see what has been found over the last 10 years and what the findings indicate (see Suga et al. 2002; Suga and Ma 2003).
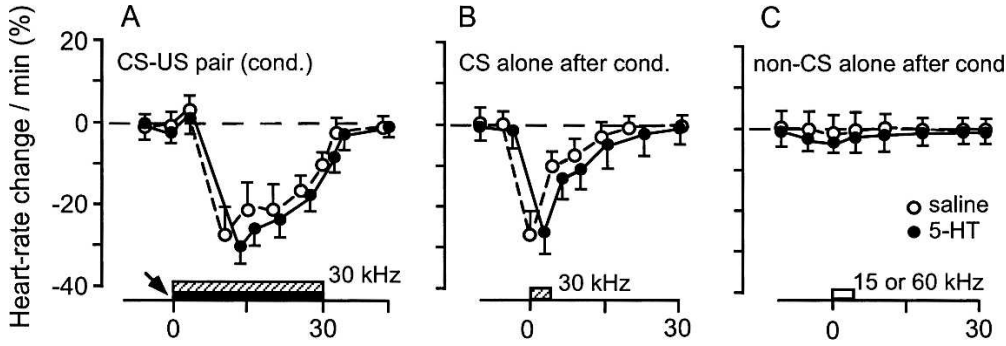

Time ( $\mathrm{min}$ ) after the onset of conditioning $(A)$ or a train of tone bursts (B and $C$ )

Figure 2. Conditioned heart-rate change. $(A)$ Heart-rate decrease elicited by the 30-min conditioning (cond.): a conditioned stimulus (CS, $30 \mathrm{kHz}$; hatched rectangle) paired with an unconditioned leg stimulus (US; filled rectangle). (B) Heart-rate decrease for the CS tone alone after the conditioning. (C) No heart-rate decrease for non-CS tones at 15 or $60 \mathrm{kHz}$ after the conditioning. A 5-HT (filled circles) or saline (open circles) solution applied to the primary auditory cortex (arrow) prior to conditioning had no effect on the conditioned autonomic response (filled circles compared with open circles; $P>0.05)$. Each symbol and bar represent a mean and standard error in the percentage of heart-rate change. Data were obtained from four bats (Ji and Suga 2007a). 


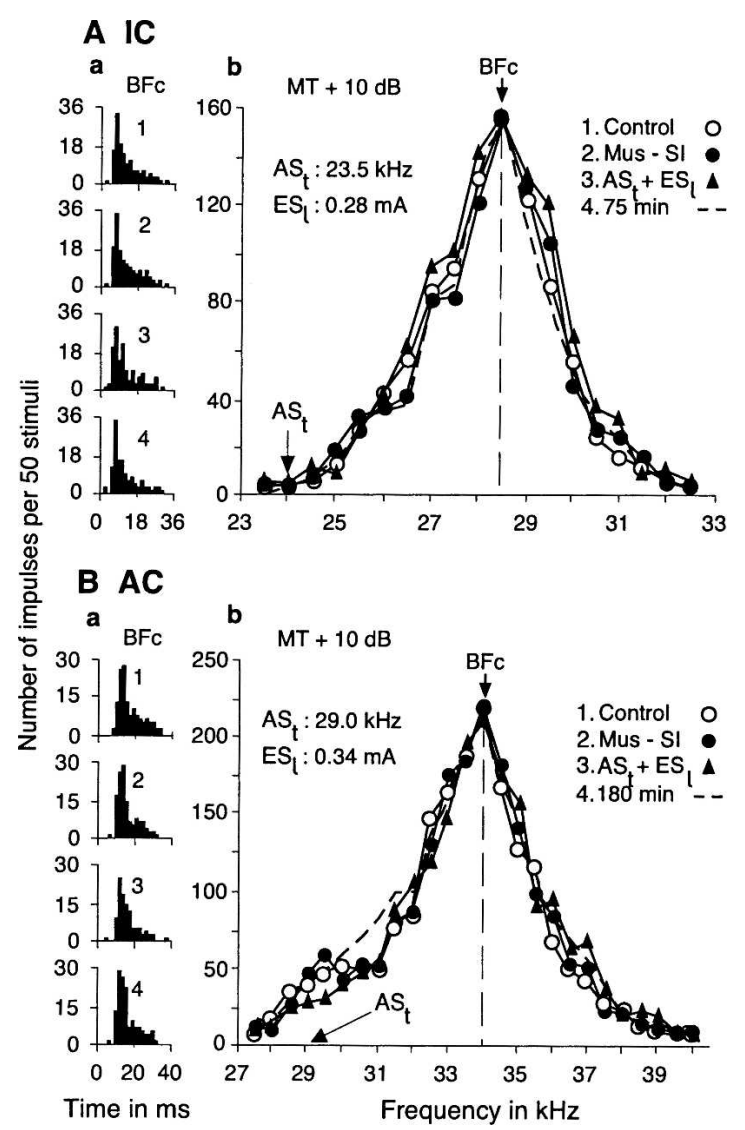

Figure 3. Bilateral inactivation of the somatosensory cortex $(\mathrm{SI})$ with $0.4 \mu \mathrm{g}$ of muscimol abolished development of the conditioningdependent $B F$ shifts of a collicular $(A)$ and a cortical $(B)$ neuron but did not change their responses $(a)$ or frequency-response curves $(b) . \mathrm{BF}_{c}, \mathrm{BF}$ in the control condition. The post-stimulus-time histograms in $a$ display the responses to tone bursts at the $\mathrm{BF}$ of a given neuron before $(1$, control condition); during bilateral muscimol applications to the surface of SIs, i.e., SI inactivation (2); immediately after conditioning under SI inactivation (3); and 75 or $180 \mathrm{~min}$ after conditioning (4). Frequency-response curves in $b$ were obtained in the above four conditions. The frequencies of conditioned acoustic stimuli $\left(\mathrm{AS}_{\mathrm{t}}\right)$ and the current of unconditioned leg stimulation $\left(\mathrm{ES}_{1}\right)$ are listed in $b$. Note that the responses $(a)$ and frequency-response curves $(b)$ of the neurons were affected by neither muscimol applied to the somtosensory cortex nor the conditioning (Gao and Suga 2000).

\section{Acknowledgment}

Our work has been supported by a research grant from the National Institute on Deafness and Other Communication Disorders (DC000175). The author thanks Sally E. Miller for editing the manuscript.

\section{Appendix}

\section{Proposed experiments}

To settle this dispute, new experimental data directly related to the disputed conclusions are necessary. Therefore, the following experiments should be performed with unanesthetized bats and/ or rodents.

1. Suga hypothesized that the MGBm does not play a role in evoking the cortical BF shift, but in evoking the nonspecific augmentation of cortical neurons. Therefore, he should perform the experiments to examine whether (a) electrical stimulation of the MGBm does not evoke cortical BF shifts; (b) this stimulation evokes nonspecific augmentation of cortical au- ditory neurons; (c) inactivation of the MGBm and PIN does not abolish the development of the conditioning-elicited cortical BF shift; and (d) this inactivation abolishes the development of nonspecific augmentation elicited by pseudoconditioning.

In rodents, it has been demonstrated that electric stimulation of the MGBv evokes the cortical BF shift (Jafari et al. 2007).

2. Weinberger doubts several neurophysiological data on which the Gao-Suga model is based. Therefore, he should perform the experiments to examine whether (a) the cortical BF shift elicited by a CS-US pair delivered at a fixed time interval is different from that elicited by the CS-US pair delivered at varied time intervals; (b) bilateral inactivation of the somatosensory cortex performed as in Gao and Suga $(1998,2000)$ abolishes the development of the conditioning-elicited collicular and cortical BF shifts; (c) collicular and cortical BF shifts are evoked by long repetitive stimulation with a single tone burst designed somewhat similarly to behaviorally relevant sounds; (d) 30-min-long repetitive stimulation with a single tone burst habituates the responses to tone bursts delivered several minutes later at frequencies several $\mathrm{kHz}$ different from the frequency of that tone burst; and (e) the BF shift of the MGBm can be elicited by auditory fear conditioning without the collicular BF shift.

\section{References}

Bakin, J.S. and Weinberger, N.M. 1996. Induction of physiological memory in the cerebral cortex by stimulation of the nucleus basalis. Proc. Natl. Acad. Sci. 93: 11219-11224.

Biordahl, T.S., Dimyan, M.A., and Weinberger, N.M. 1998. Induction of long-term receptive field plasticity in the auditory cortex of the waking guinea pig by stimulation of the nucleus basalis. Behav. Neurosci. 112: 467-479.

Chowdhury, S.A. and Suga, N. 2000. Reorganization of the frequency map of the auditory cortex evoked by cortical electrical stimulation in the big brown bat. J. Neurophysiol. 83: 1856-1863.

Edeline, J.M., Hars, B., Hennevin, E., and Cotillon, N. 2002. Muscimol diffusion after intracerebral microinjections: A reevaluation based on electrophysiological and autoradiographic quantifications. Neurobiol. Learn. Mem. 78: 100-124.

Gao, E. and Suga, N. 1998. Experience-dependent corticofugal adjustment of midbrain frequency map in bat auditory system. Proc. Natl. Acad. Sci. 95: 12663-12670.

Gao, E. and Suga, N. 2000. Experience-dependent plasticity in the auditory cortex and the inferior colliculus of bats: Role of the corticofugal system. Proc. Natl. Acad. Sci. 97: 8081-8086.

Jafari, M.R., Zhang, Y., and Yan, J. 2007. Multiparametric changes in the receptive field of cortical auditory neurons induced by thalamic activation in the mouse. Cereb. Cortex 17: 71-80.

Ji, W. and Suga, N. 2003. Development of reorganization of the auditory cortex caused by fear conditioning: Effect of atropine. $J$. Neurophysiol. 90: 1904-1909.

Ji, W. and Suga, N. 2007a. Serotonergic modulation of plasticity of the auditory cortex elicited by fear conditioning. J. Neurosci. 27: 4910-4918.

Ji, W. and Suga, N. 2007b. Cortical tone-specific plasticity elicited by conditioning and cortical non-specific plasticity elicited by pseudoconditioning depend on different neuromodulators. Prog. No. 174.4. In 2007 Neuroscience Meeting Planner. Society for Neuroscience, Washington DC.

Ji, W., Gao, E., and Suga, N. 2001. Effects of acetylcholine and atropine on plasticity of central auditory neurons caused by conditioning in bats. J. Neurophysiol. 86: 211-225.

Ji, W., Suga, N., and Gao, E. 2005. Effects of agonists and antagonists of NMDA and Ach receptors on plasticity of bat auditory system elicited by fear conditioning. J. Neurophysiol. 94: 1199-1211.

Kilgard, M.P. and Merzenich, M.M. 1998. Cortical map reorganization enabled by nucleus basalis activity. Science 279: 1714-1718.

Ma, X. and Suga, N. 2001. Plasticity of bat's central auditory system evoked by focal electric stimulation of auditory and/or somatosensory cortices. J. Neurophysiol. 85: 1078-1087.

$\mathrm{Ma}, \mathrm{X}$. and Suga, N. 2003. Augmentation of plasticity of the central auditory system by the basal forebrain and/or somatosensory cortex. I. Neurophysiol. 89: 90-103.

Ma, X. and Suga, N. 2004. Lateral inhibition for center-surround 
reorganization of the frequency map of bat auditory cortex. $J$. Neurophysiol. 92: 3192-3199.

$\mathrm{Ma}, \mathrm{X}$. and Suga, N. 2005. Long-term cortical plasticity evoked by electric stimulation and acetylcholine applied to the auditory cortex. Proc. Natl. Acad. Sci. 102: 9335-9340.

Sakai, M. and Suga, N. 2001. Plasticity of the cochleotopic (frequency) map in specialized and nonspecialized auditory cortices. Proc. Natl. Acad. Sci. 98: 3507-3512.

Sakai, M. and Suga, N. 2002. Centripetal and centrifugal reorganizations of frequency map of the auditory cortex in gerbils. Proc. Natl. Acad. Sci. 99: 7108-7112.

Suga N. 2008. Role of corticofugal feedback in hearing. J. Comp. Physiol. A: Neuroethol., Sensory, Neural, Behav. Physiol. 194: 169-183.

Suga, N. and Ma, X. 2003. Multiparametric corticofugal modulation and plasticity in the auditory system. Nat. Rev. Neurosci. 4: 783-794.

Suga, N., Gao, E., Zhang, Y., Ma, X., and Olsen, J.F. 2000. The corticofugal system for hearing: Recent progress. Proc. Natl. Acad. Sci. 97: 11807-11814.

Suga, N., Xiao, Z., Ma, X., and Ji, W. 2002. Plasticity and corticofugal modulation for hearing in adult animals. Neuron 36: 9-18.

Villa, A.E., Rouiller, E.M., Simm, G.M., Zurita, P., de Ribaupierre, Y., and de Ribaupierre, F. 1991. Corticofugal modulation of the information processing in the auditory thalamus of the cat. Exp. Brain Res. 86: $506-517$.

Weinberger, N.M. 1998. Physiological memory in primary auditory cortex: Characteristics and mechanisms. Neurobiol. Learn. Mem. 70: $226-251$.

Weinberger, N.M. 2004. Specific long-term memory traces in primary auditory cortex. Nat. Rev. Neurosci. 5: 279-290.

Weinberger, N.M. 2007. Associative representational plasticity in the auditory cortex: A synthesis of two disciplines. Learn. Mem. 14: 1-16.

Wu, Y. and Yan, J. 2007. Modulation of the receptive fields of midbrain neurons elicited by thalamic electrical stimulation through corticofugal feedback. J. Neurosci. 27: 10651-10658.

Xiao, Z. and Suga, N. 2002. Reorganization of the cochleotopic map in the bat's auditory system by inhibition. Proc. Natl. Acad. Sci. 99: $15743-15748$.

Yan, J. and Ehret, G. 2001. Corticofugal reorganization of the midbrain tonotopic map in mice. Neuroreport 12: 3313-3316.

Yan, J. and Ehret, G. 2002. Corticofugal modulation of midbrain sound processing in the house mouse. Eur. J. Neurosci. 16: 119-128.

Yan, J. and Zhang, Y. 2005. Sound-guided shaping of the receptive field in the mouse auditory cortex by basal forebrain activation. Eur. J. Neurosci. 21: 563-576.

Zhang, Y. and Suga, N. 2005. Corticofugal feedback for collicular plasticity evoked by electric stimulation of the inferior colliculus. $J$. Neurophysiol. 94: 2676-2682.

Zhang, Y., Hakes, J.J., Bonfield, S.P., and Yan, J. 2005. Corticofugal feedback for auditory midbrain plasticity elicited by tones and electrical stimulation of basal forebrain in mice. Eur. J. Neurosci. 22: 871-879.

Received January 28, 2008; accepted in revised form February 1, 2008. 


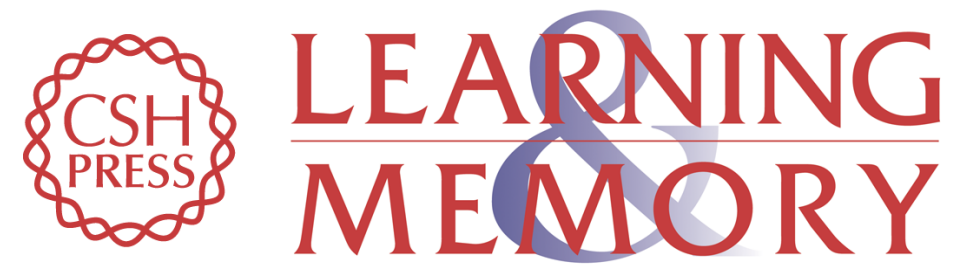

\section{The neural circuit for tone-specific plasticity in the auditory system elicited by conditioning}

Nobuo Suga

Learn. Mem. 2008, 15:

Access the most recent version at doi:10.1101//m.791408

References This article cites 33 articles, 12 of which can be accessed free at: http://learnmem.cshlp.org/content/15/4/198.full.html\#ref-list-1

License

Email Alerting Receive free email alerts when new articles cite this article - sign up in the box at the Service top right corner of the article or click here. 